Abstract Monocrystalline titanium dioxide $\left(\mathrm{TiO}_{2}\right)$ microspheres support two orthogonal magnetic dipole modes at terahertz $(\mathrm{THz})$ frequencies due to strong dielectric anisotropy. For the first time, we experimentally detected the splitting of the first Mie mode in spheres of radii $10-20 \mu \mathrm{m}$ through near-field time-domain $\mathrm{THz}$ spectroscopy. By fitting the Fano lineshape model to the experimentally obtained spectra of electric field detected by the sub-wavelength aperture probe, we found that the magnetic dipole resonances in $\mathrm{TiO}_{2}$ spheres have narrow linewidths of only tens of gigahertz. Anisotropic $\mathrm{TiO}_{2}$ microresonators can be used to enhance the interplay of magnetic and electric dipole resonances in emerging $\mathrm{THz}$ all-dielectric metamaterial technology.

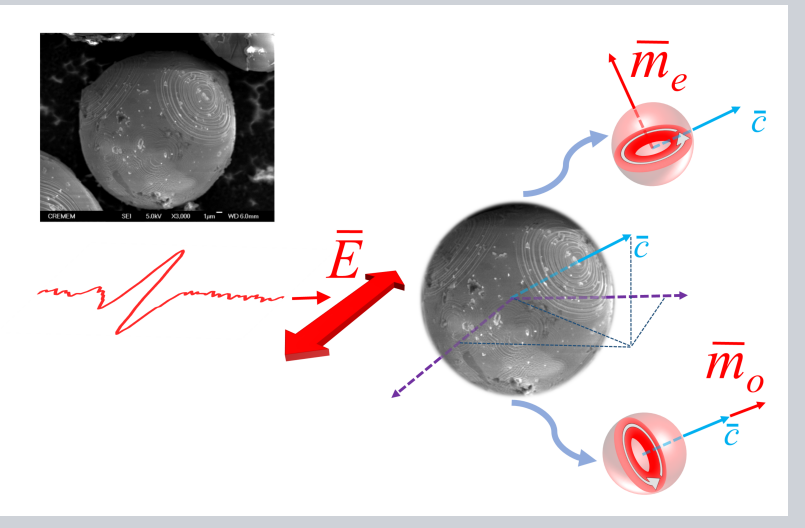

\title{
Splitting of magnetic dipole modes in anisotropic $\mathrm{TiO}_{2}$ micro-spheres.
}

\author{
Irina Khromova ${ }^{1-3 *}$, Petr Kužel ${ }^{4}$, Igal Brener ${ }^{5,6}$, John L. Reno ${ }^{5,6}$, U-Chan Chung Seu ${ }^{7}$, \\ Catherine Elissalde $^{7}$, Mario Maglione ${ }^{7}$, Patrick Mounaix ${ }^{8}$, and Oleg Mitrofanov ${ }^{2,5}$
}

\section{Introduction}

Sub-wavelength dielectric resonators are the building blocks of all-dielectric metamaterials [1-5] and metasurfaces [6$8]$ - artificially created materials with engineerable effective dielectric permittivity and magnetic permeability or local impedance. The required macroscopic magnetic response [9] in such non-magnetic structures is realised through arranging sub-wavelength dielectric resonators, sustaining magnetic dipole modes [10], in an array.

Spectrally overlapping magnetic and electric dipole modes in a dielectric resonator create a Huygens source [11], which can be a base for reflectionless all-dielectric metasurfaces. To achieve such overlapping, either substrate effects $[12,13]$ or geometrical anisotropy $[7,8]$ can be employed. Material anisotropy, such as in mono-crystalline $\mathrm{TiO}_{2}$, in geometrically-isotropic dielectric resonators can lead to similar effects. Moreover, anisotropic dielectric resonators give an additional degree of freedom for collective resonant effects [14].

To form a metamaterial, resonant 'meta-atoms' have to be packed at a scale significantly below $\lambda / 2$, where $\lambda$ is the resonance wavelength in free space. To sustain electromagnetic resonances at a sub-wavelength scale sufficient for the effective medium approximation in all-dielectric metama- terials, constituent resonators have to be made of materials with high values of dielectric permittivity.

At optical frequencies, it is hard to find such materials and, thus, to achieve strong resonant confinement: silicon resonators with dielectric permittivity of $\varepsilon=16$ resonating at a wavelength of $\lambda=800 \mathrm{~nm}$ are only $\approx \lambda / 4$ in size [15], where $\lambda$ is the resonance wavelength in free space. This makes it barely possible to pack such resonators at a sub-wavelength scale (with pitch significantly below $\lambda / 2)$ to form a metamaterial or a metasurface. In the terahertz $(\mathrm{THz})$ frequency range, high values of dielectric permittivity are not unusual. $\mathrm{TiO}_{2}$ micro-spheres with $\varepsilon \approx 100$ confine electromagnetic energy resonantly within a space with linear dimensions on the order of $\lambda / 10[16,17]$. Allowing for sub-wavelength packing, they are a promising foundation for emerging all-dielectric terahertz metamaterials $[18,19]$.

$\mathrm{TiO}_{2}$ can be highly anisotropic [20]: the reported ordinary and extraordinary permittivities of bulk rutile $\mathrm{TiO}_{2}$ in the THz frequency range are $\varepsilon_{o} \approx 80$ to $\varepsilon_{e} \approx 150$. Thus, the orientation of mono-crystalline $\mathrm{TiO}_{2}$ micro-spheres critically affects their spectroscopic properties. In addition, $\mathrm{TiO}_{2}$ micro-spheres may show significant variability in morphology, crystallinity, density, shape and surface roughness. So far, the $\mathrm{THz}$ spectral signatures of anisotropic $\mathrm{TiO}_{2}$ micro-spheres have not be measured.

\footnotetext{
${ }^{1}$ Department of Physics, King's College London, Strand, London WC2R 2LS, UK $\quad{ }^{2}$ Department of Electronic \& Electrical Engineering, UCL, London, WC1E 7JE, UK ${ }^{3}$ ITMO University, St. Petersburg 199034, Russia ${ }^{4}$ Institute of Physics, Academy of Sciences of the Czech Republic, 18221 Prague 8, Czech Republic ${ }^{5}$ Center for Integrated Nanotechnologies, Sandia National Laboratories, NM 87185, USA ${ }^{6}$ Sandia National Laboratories, Albuquerque, New Mexico 87185, USA 7 ICMCB, University of Bordeaux, CRNS, UPR 9048, 87 Avenue du Docteur Schweitzer, 33608 Pessac, France ${ }^{8}$ LOMA, University of Bordeaux, CNRS, UMR 5798, 351 Cours de la Libration, 33405 Talence, France

* Corresponding author: e-mail: irina.khromova@kcl.ac.uk
} 

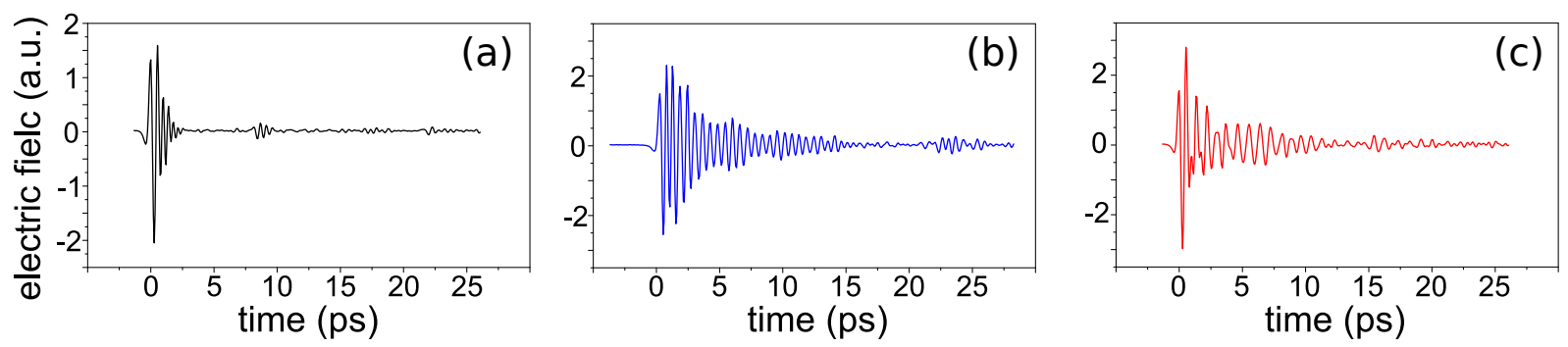

Figure 1 (a): Reference waveform, and $(b, c)$ waveforms measured when $\mathrm{TiO}_{2}$ spheres of radii $r=10.7 \mu \mathrm{m}$ and $r=15.5 \mu \mathrm{m}$ respectively, are placed in front of the aperture probe. The orientation of the smaller sphere is such that only one - the ordinary - magnetic resonance mode is excited $(b)$. Both the ordinary and the extraordinary modes are excited in the larger sample $(c)$.

In this paper, we propose and demonstrate a technique for characterising sub-wavelength mono-crystalline $\mathrm{TiO}_{2}$ micro-spheres using near-field THz microscopy [17,21,22]. For the first time, we experimentally detected the splitting of the lowest resonant Mie mode into two distinct magnetic-dipole eigenmodes due to the pronounced dielectric anisotropy of the micro-sphere material. We demonstrate that the intrinsic properties of high-permittivity anisotropic resonators can be extracted despite the undetermined orientation of the crystallographic axes of the constituent anisotropic material. We found that the linewidths of the observed $\mathrm{THz}$ resonances are of the order of tens of gigahertz. They also vary significantly among individual micro-spheres measured, indicating that that the fabrication process has a critical effect on the resonators' properties.

\section{Methods}

$\mathrm{TiO}_{2}$ spherical particles, $10-40 \mu \mathrm{m}$ in diameter, are fabricated by spraying $\mathrm{TiO}_{2}$ nano-crystal suspension into a flame and subsequent annealing at $1400 \mathrm{C}^{\circ}$. The annealing process melts poly-crystalline particle clusters into monocrystal $\mathrm{TiO}_{2}$ spheres. X-ray diffraction (XRD) analysis confirmed that the main constituent is $\mathrm{TiO}_{2}$ in the rutile tetragonal phase. It also identified monoclinic $\mathrm{Ti}_{3} \mathrm{O}_{5}$ as a minority phase. A scanning electron microscopy (SEM) image of one of the studied samples is shown in the title Figure. SEM analysis showed small voids and inclusions within cracked spheres and also revealed that some micro-spheres contain several large mono-crystal domains.

To investigate Mie resonances, single spheres were attached to a $12.5 \mu \mathrm{m}$-thick polyethylene substrates and positioned within several micrometers from a $\mathrm{THz}$ near-field probe comprised of a $10 \mu \mathrm{m} \times 10 \mu \mathrm{m}$ aperture in a gold screen and a photoconductive antenna detector behind the screen [21]. The samples were illuminated at normal incidence to the screen plane by a linearly-polarized ( $x$-axis) single-cycle $\mathrm{THz}$ pulses generated using a ZnTe crystal. The photoconductive $\mathrm{THz}$ antenna detected the $E_{x}$ component of $\mathrm{THz}$ field coupled through the aperture. The nearfield response for each sphere was obtained for different angles of rotation in the $x y$-plane within the spectral range of $0.5-2.5 \mathrm{THz}$.
Full-wave numerical versions of the experiments were performed using the time-domain solver of the CST Microwave Studio $^{\mathrm{TM}}$. We modelled the response of anisotropic spheres in the range of $0-2.5 \mathrm{THz}$ taking the substrate and the near-field probe into account as in [22]. We set the finite size of the gold screen as $500 \mu \mathrm{m}$, which is more than 10 times the diameter of any of the measured samples. We used hexahedral mesh cells with linear sizes varying from 0.3 to $10 \mu \mathrm{m}$.

\section{Results and Discussion}

\subsection{Mie resonances}

Two non-degenerate magnetic dipole modes are expected to be excited in a mono-crystalline $\mathrm{TiO}_{2}$ sphere when its optical axis ( $c$ crystallographic axis) is not aligned with or not strictly perpendicular to the incident $\mathrm{THz}$ field vector (see title Figure). In other words, when the incident electric field has both components parallel and perpendicular to the optical axis of $\mathrm{TiO}_{2}$, it excites two orthogonal magnetic modes: one at a higher frequency (ordinary mode; electric field polarisation plane inside the sphere is perpendicular to the optical axis of $\mathrm{TiO}_{2}$ ) and one at a lower frequency (extra-ordinary mode; the electric field polarisation plane contains the optical axis of $\mathrm{TiO}_{2}$ ), see title Figure.

Figure 1 shows typical waveforms of the detected $\mathrm{THz}$ field coupled through the aperture of the probe. The incident $\mathrm{THz}$ pulse sampled by the sub-wavelength aperture probe is shown in Figure 1(a). Measuring the timedomain response of the micro-spheres, we observed two types of resonant waveforms: Fig. 1(b) shows harmonic oscillation of the field that lasts over 20 ps; Fig.1(c) presents an amplitude-modulated oscillation of the field. The first case corresponds to the resonant response of a sample due to the excitation of a single magnetic dipole mode. In the second case, the 'beating' indicates the excitation of two resonances simultaneously. The splitting of the modes is discussed in detail in Section 3.4.

The field oscillations in the waveform in Fig.1(b) represent the enhancement of the electric field within a narrow frequency range. The Fourier transform of the waveform Fig.1(b), measured for the sample with radius $r=10.7 \mu \mathrm{m}$ (sample \# 1) and normalized to the corresponding reference 

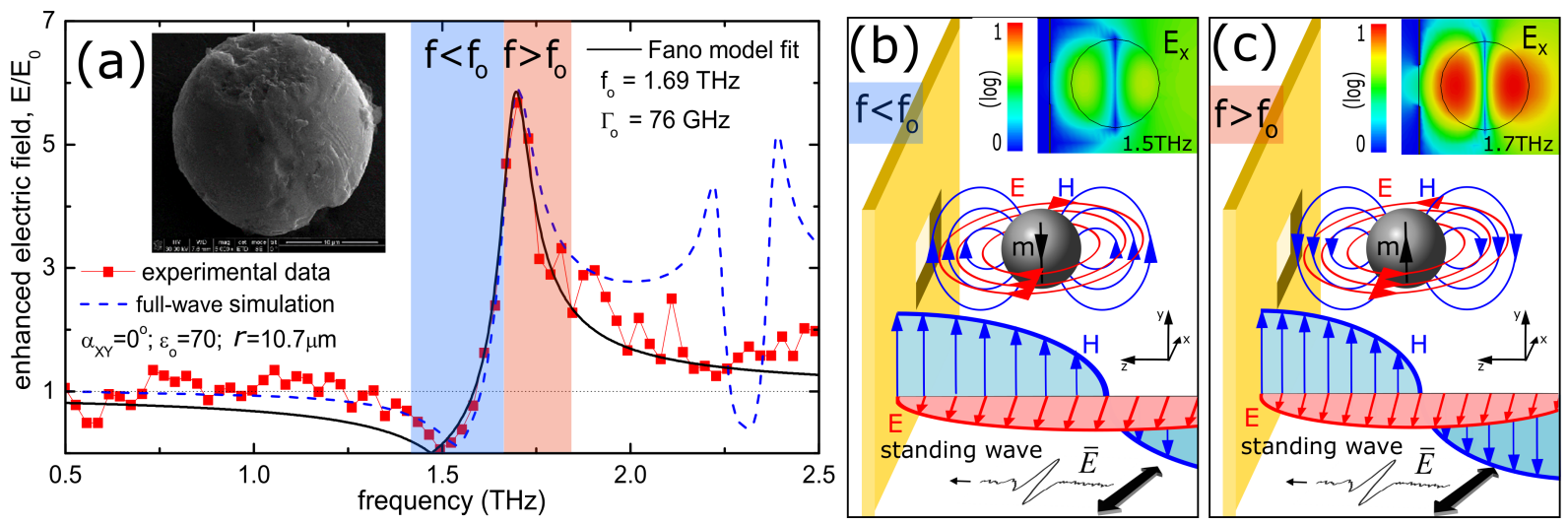

Figure 2 (a): Experimentally obtained (red curve and squares) and numerically simulated (blue dashed curve) enhanced electric field spectrum of an anisotropic $\mathrm{TiO}_{2}$ sphere of radius $r=10.7 \mu \mathrm{m}$ (measured using SEM: inset image) with its main axis oriented perpendicularly to the incident $\mathrm{THz}$ pulse polarization plane. The black curve is a Fano model fit with linewidth $\Gamma_{o}=76 G H z$ and resonance frequency $f_{o}=1.69 \mathrm{THz} ;(b)$ and $(c)$ : Schematic spatial distributions of magnetic (blue) and electric (red) fields excited in magnetic dipole modes supported by $\mathrm{TiO}_{2}$ spheres at frequencies below $(b)$ and above $(c)$ the resonance frequency. The insets show the corresponsing numerically calculated maps of the $x$-component of the electric field. At each frequency, the sphere is excited by a corresponding standing wave formed due to reflections from the metallic detector. The samples are located between the ground plane of the detector and the first node of the magnetic field, $H_{y}$. The field leaking through the aperture (and detected by the THz photoconductive antenna in case of $E_{x}$ ) is a superposition of the fields induced in the resonator and the background field corresponding to the incident $\mathrm{THz}$ beam.

spectrum (Fourier transform of the waveform in Fig.1(a)), is shown in Fig.2(a).

We adjusted the position of the resonance peak in fullwave numerical simulations for sample \# 1 (blue dashed curve in Fig.2 (a) to fit the experimental data by varying the ordinary permittivity value of $\mathrm{TiO}_{2}$. We found its value to be equal to $\varepsilon_{o}=70$. Since the estimation of dimensions for the rest of the samples was less accurate (see details in Table 1), we considered this value of ordinary permittivity as a reference for all the measured $\mathrm{TiO}_{2}$ micro-spheres. We set the dielectric loss tangent of $\mathrm{TiO}_{2}$ to $\tan \delta=0.02$, which is consistent with previous measurements for $\mathrm{TiO}_{2}$ [20].

The spectra of enhanced electric field have Fano-like lineshapes in the vicinities of magnetic dipole modes (Fig.2(a)). Fitting the analytical function describing a Fano resonance to the experimental data allowed us to extract the intrinsic properties of the studied dielectric microresonators.

Higher frequency resonances, including electric dipole modes, were observed in the simulations. However, their signatures in our experimental data are less pronounced. We attribute this to the reduced sensitivity of the experimental system at higher frequencies.

\subsection{Origin of the Fano lineshape of the electric field enhanced by a dielectric micro-resonator}

In this Section, we discuss the origin of the observed Fano lineshapes in the near-field spectra of the dielectric microspheres.

As the $\mathrm{THz}$ pulses are incident normally to the metallic plane of the near-field probe, the sphere can be considered excited by standing waves formed by incident and re- flected plane waves at each frequency of the $\mathrm{THz}$ pulse. The field coupled through the aperture is a superposition of the incident $\mathrm{THz}$ field and the resonant field induced in the sphere by the standing wave. Figures $2(b),(c)$ illustrate the resonant electric and magnetic fields excited in a sub-wavelength-sized sphere and the corresponding standing wave excitation. Therefore, the shape of the detected spectrum can be described by the Fano model $[23,24]$ :

$$
\frac{E}{E_{0}}=\sqrt{\frac{\left(q_{o, e} \times \Gamma_{o, e} / 2+f-f_{o, e}\right)^{2}}{\left(\left(\Gamma_{o, e} / 2\right)^{2}+\left(f-f_{o, e}\right)^{2}\right)}},
$$

where $f_{o, e}$ are the frequencies of the ordinary and extraordinary resonance modes of a sphere (see Section 3.4), $\Gamma_{o, e}$ are the lindewidths of the corresponding peaks, $q_{o, e}$ are the Fano amplitude parameters. The Fano models fitted to the measured spectra are shown as black solid/dashed curves in Fig. 2(a),4, and 5.

At frequencies below the resonance frequency, the electric field of the magnetic dipole mode (Fig. 2(b)) interferes destructively with the standing wave (between the ground plane and the first node of the magnetic field $H_{y}$ ) in a narrow frequency range. In the measured spectra, we observed characteristic amplitude dips at frequencies slightly below the resonance frequencies of the magnetic dipole modes (highlighted in blue in Fig. 2(a)).

At frequencies above the resonance frequency, the resonant mode field lags the excitation field, leading to the constructive interference of the modal fields with the standing wave (Fig. 2(c)). Corresponding field enhancement peaks were observed in all the measured spectra (highlighted in red in Fig. 2(a)). 
The Fano lineshape of the resonance modes is closely connected to the detection process itself. Our simulations indicate that the Fano lineshape of the detected enhanced near-field progressively changes as the distance between the sphere and the metallic $\mathrm{THz}$ probe increases (see insets in Fig. 3(b)).

In the presence of the metallic plate, the polarity of the observed Fano shape depends on the symmetry of the studied mode and the place where it is measured. In case of the magnetic dipole mode discussed here, the 'polarity' is reversed at the front surface of the sphere (further edge with respect to the probe), where the resonant electric field has an opposite phase. Therefore, a reversed spectrum - constructive interference below the resonance and destructive interference above the resonance - is expected to be observed at this position. Note that a different near-field detection scheme, including e.g. a scattering-type near-field probe, would be required for such an experiment.

At each frequency, the phase relation between the incident field (standing waves) and the magnetic resonance mode is defined by the position of the sphere with respect to the nodes of the standing wave.

In our experiment, the studied spheres were always placed at distances of several micrometres from the probe: thus, they were always between the ground plane and the first node of the $H_{y}$ component of the incident magnetic field at the resonance frequency (always below $\lambda_{\max } / 2=$ $60 \mu \mathrm{m})$.

\subsection{Intrinsic properties of dielectric micro-resonators}

The intrinsic characteristics of Mie modes, i.e. resonance frequencies and linewidths, are defined by the geometrical and material properties of the resonator. The corresponding experimental values may be also influenced by the presence of the substrate and the metallic probe (ground-plane effect). The aim of this Section is to characterize these dependences.

Unlike metallic micro-objects [22] or dielectric resonators made of low-permittivity materials [26], the resonance modes of the studied micro-spheres are confined within the resonators due to the high dielectric permittivity of $\mathrm{TiO}_{2}$. Therefore, the influence of the low-permittivity substrate (polyethylene) on the observed resonance properties is minimal [27].

To address the effect of the metallic probe on the resonant properties of the samples, we numerically simulated spheres suspended in free space, and spheres placed on top of a perfect electric conductor, which emulated the conditions of our experiment. The resonance frequencies in both cases were found using the far-field scattering cross-section spectra. Figure 3(a) shows the resonant frequencies calculated numerically for identical $\mathrm{TiO}_{2}$ spheres suspended in free space (blue solid curve) and placed on top of a metallic plane (blue dashed curve). The blue squares represent the resonance frequency obtained from fitting a Fanoresonance curve (Eq. 1) to the data obtained via numerical
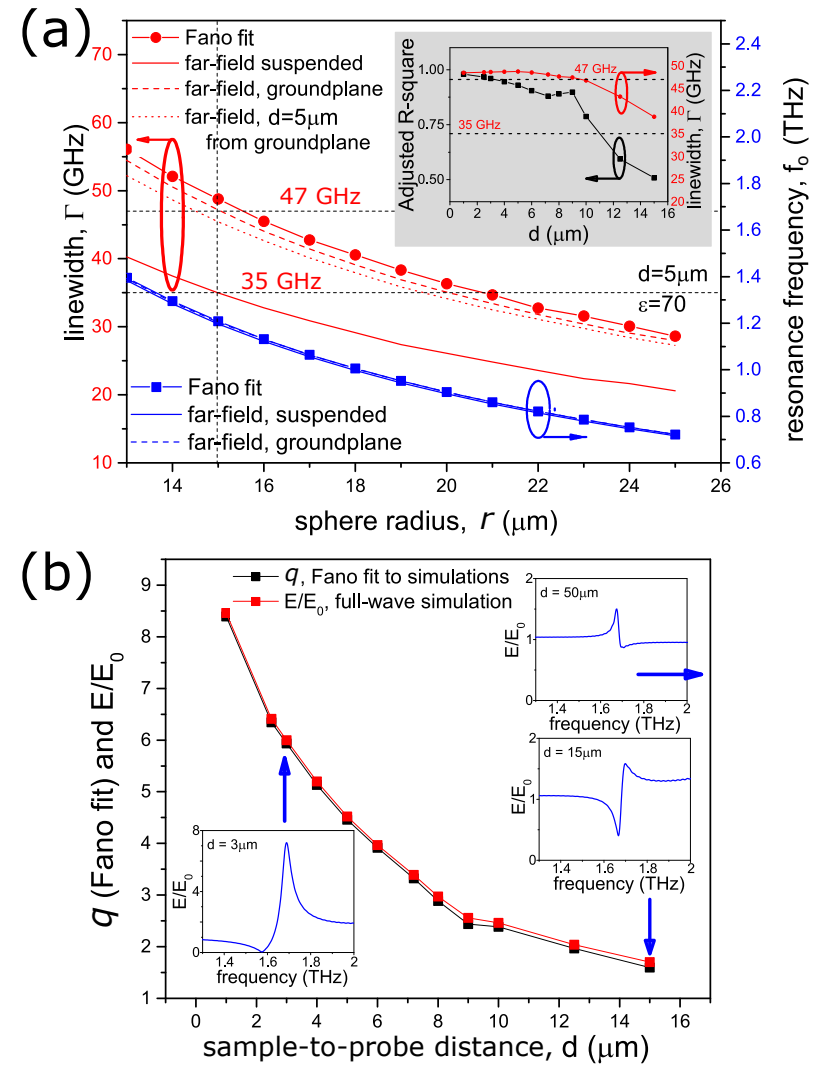

Figure 3 (a): Linewidths (red, left axis) and magnetic dipole mode resonance frequencies (blue, right axis) of a sphere $(\varepsilon=$ 70) suspended in free space (solid), placed on top of a perfect electric conductor substrate (dashed) and at a distance of $d=5 \mu \mathrm{m}$ from the latter (dotted) plotted against the sphere's radius $r$. These results were extracted from far-field numerical simulations. Red circles and blue squares are linewidths and resonance frequencies extracted from Fano model fitting applied to the spectra obtained by near-field numerical experiment (same sphere placed at a distance of $d=5 \mu \mathrm{m}$ from the probe). Inset: dependence of the linewidth and of the quality of the Fano model fitting (adjusted R-square [25]) on the sample-to-probe distance. (b): Enhancement factor $E / E_{0}$ and Fano model amplitude $q$ for a sphere $(r=15 \mu \mathrm{m}, \varepsilon=70)$ versus sample-to-probe distance. The insets show the change in the Fano-like lineshape of the enhanced electric field as the sample is removed from the $\mathrm{THz}$ probe.

near-field experiment described in Sec. 2. The differences between the three resonance frequencies for all considered radii of the micro-spheres are negligible. The resonance frequency detected in the experiment, therefore, can be considered as the intrinsic frequency of the $\mathrm{TiO}_{2}$ sphere despite the presence of the ground plane of the probe.

In contrast to the resonance frequency, the linewidth of the sphere placed on top of a metallic plane (red dashed line in Fig. 3(a)), is noticeably broadened, by $10-15 \mathrm{GHz}$, as compared to the suspended case (red solid line). The linewidths extracted through Fano-fitting of the data obtained in our near-field numerical experiments (red squares) were confirmed to have values similar to those obtained in 


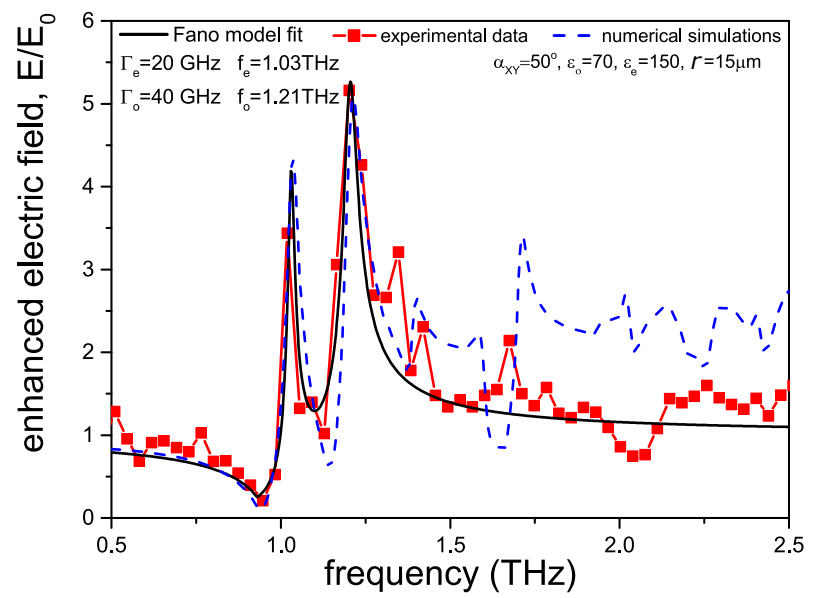

Figure 4 Experimentally obtained (red curve and squares) and numerically simulated (blue dashed curve) enhanced electric field spectrum of an anisotropic $\mathrm{TiO}_{2}$ sphere of radius $r=15 \mu \mathrm{m}$ (estimated from numerical simulations using ordinary permittivity of $\varepsilon_{o}=70$ ) with its main axis oriented at an angle $\alpha_{X Y}=50^{\circ}$ to the incident linearly polarized $\mathrm{THz}$ light. The black curve is a superposition of two Fano model fits.

far-field simulations for a sphere sitting on top of and at a $5 \mu \mathrm{m}$ distance away from a metallic plane.

For a sphere placed within $\approx 10 \mu \mathrm{m}$ from the metallic plane, the linewidth of the Mie resonance of the sphere, extracted through Fano-fitting of its numerical near-field spectra, varies by less than $2 \mathrm{GHz}$, which is demonstrated in the inset in Fig. 3(a) (red circles). At distances larger than $d \approx 10 \mu \mathrm{m}$, the proposed Fano-fitting method becomes less reliable due to the drop in the resonant field enhancement at larger sample-to-probe distances (inset in Fig. 3(a), black squares). Thus, we conclude that the linewidth extracted by fitting the Fano curve to the measured near-field spectra, can be considered independent of the sample-to-probe distance for $d<10 \mu \mathrm{m}$.

In contrast, the amplitude $q$ of the Fano resonance changes significantly even at small sample-to-probe distance (Fig. 3(b)). This is due to the strong near-field confinement of the resonant modes and the interaction of the resonant near-field with the standing waves formed behind the ground plane of the detector (see Sec. 3.2)). We note that the ratio of the resonance frequency to the resonance linewidth, or the quality factor of the resonator, is independent of sphere size. We can conclude that while the Fano lineshape (determined essentially by the amplitude $q$ ) depends strongly on experimental conditions, the measured resonance frequency (and to a large extent also the linewidth for $d<10 \mu \mathrm{m}$ ) do correspond to intrinsic parameters of the observed Mie resonances.

\subsection{Splitting of magnetic resonance modes due to anisotropy in mono-crystalline $\mathrm{TiO}_{2}$}

Studying different orientations of the samples in the $x y$ plane, we observed two types of spectral signatures: the

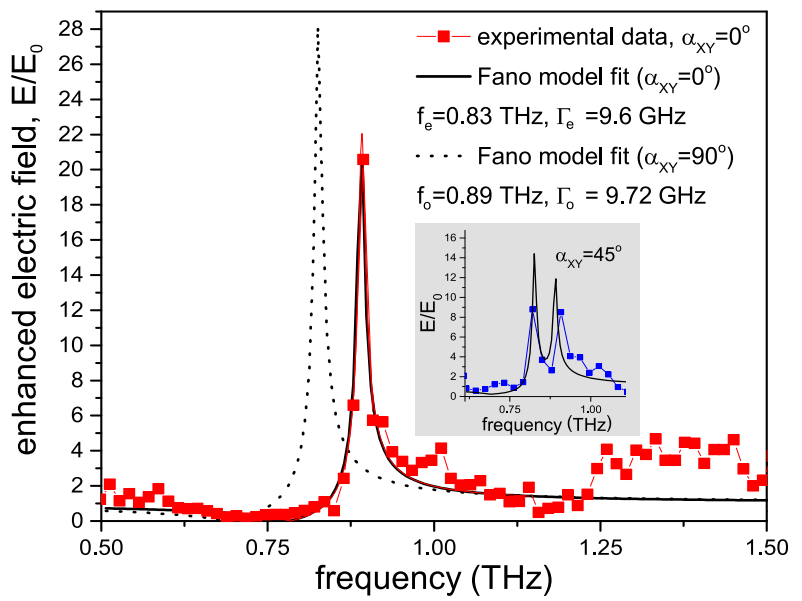

Figure 5 Experimentally obtained (red/blue curve and squares) enhanced electric field spectrum of an anisotropic $\mathrm{TiO}_{2}$ sphere of radius $r=19.5 \mu \mathrm{m}$ with its main axis oriented at $\alpha_{X Y}=0^{\circ}, 45^{\circ}$ with respect to the incident linearly polarized THz light. The black solid and dashed curves are Fano models fitted to the experimental data and taking the orientation of the sample into account $\left(\alpha_{X Y}=0^{\circ}, 45^{\circ}\right.$ and $\left.90^{\circ}\right)$.

excitation of a single (either the ordinary or the extraordinary) magnetic dipole resonance or of two resonances simultaneously. The ordinary magnetic dipole modes in $\mathrm{TiO}_{2}$ spheres have higher frequency than the extraordinary magnetic dipole modes: the electric field of the ordinary magnetic dipole mode lies in the plane where spheres' effective permittivity is $\varepsilon_{o}$, which is lower compared to the averaged effective permittivity of $\approx\left(\varepsilon_{o}+\varepsilon_{e}\right) / 2$ 'seen' by the extraordinary magentic dipole mode.

The spectra in Fig. 2(a) reflect the excitation of the higher-frequency ordinary magnetic dipole mode of a sphere of radius $r=10.7 \mu \mathrm{m}$. In this case, the $c$-axis of $\mathrm{TiO}_{2}$ is strictly perpendicular to the incident field polarization plane, and the electric field of the magnetic dipole mode experiences only the ordinary component of the dielectric tensor, $\varepsilon_{o}$.

Figure 4 illustrates the spectrum of the sphere of radius $r=15 \mu \mathrm{m}$, in which the ordinary and extraordinary magnetic dipole modes are excited simultaneously. The ratio of the peak amplitudes is related to the in-plane angle $\alpha_{X Y}$ between the THz field polarization plane and the optical axis in the sphere. In the case of Fig. 4, its value was found to be equal to $50^{\circ}$ via full-wave numerical simulations. Figure 5 shows the ordinary magnetic resonance mode for a sample of radius $r=19.5 \mu \mathrm{m}$. The $c$-axis in this case is perpendicular to the field polarisation plane. In the same sample oriented at $\alpha_{X Y}=45^{\circ}$, both magnetic dipole modes were detected (inset in Fig. 5).

The linewidths of the resonances extracted using the Fano profile fit (Eq. 1) to the experimental near-field data are presented in Table 1. As discussed earlier in Sec. 3.3, these near-field data give an upper limit for the intrinsic linewidth of such resonators. Thus, any dielectric resonators characterised using the present method are expected to exhibit even narrower resonances in free space. 
I. Khromova et al.: Splitting of magnetic dipole modes in anisotropic $\mathrm{TiO}_{2}$ micro-spheres

\begin{tabular}{cccccccccccc}
$\#$ & $r(\mu \mathrm{m})$ & $f_{e}(\mathrm{THz})$ & $f_{o}(\mathrm{THz})$ & $\varepsilon_{e}$ & $\varepsilon_{o}$ & $\Gamma_{e}(\mathrm{GHz})$ & $\Gamma_{o}(\mathrm{GHz})$ & $q_{e}$ & $q_{o}$ & $Q F_{e}$ & $Q F_{o}$ \\
\hline 1 & $10.7 \pm 0.5$ & - & $1.69 \pm 0.058$ & - & $70 \pm 4.9$ & - & 76 & - & 5.8 & - & 22 \\
2 & $11.5 \pm 0.64^{*}$ & $1.41 \pm 0.058$ & $1.59 \pm 0.058$ & $125 \pm 10.5$ & $70^{*}$ & 72 & 64 & 3.63 & 3.75 & 20 & 25 \\
3 & $15 \pm 0.64^{\dagger}$ & $1.03 \pm 0.058$ & $1.21 \pm 0.058$ & $150 \pm 17$ & $70^{\dagger}$ & 20 & 40 & 10 & 7.81 & 52 & 30 \\
4 & $19.5 \pm 0.32^{*}$ & $0.83 \pm 0.029$ & $0.89 \pm 0.029$ & $130 \pm 9.2$ & $70^{*}$ & 9.6 & 9.72 & 28.36 & 22.04 & 87 & 92 \\
\hline
\end{tabular}

Table 1 Measured and simulated characteristics of the studied $\mathrm{TiO}_{2}$ spheres, their Fano-model fitting parametres, and the estimated quality factors $Q F_{o, e}$. The errors in $f_{o, e}$ and $\varepsilon_{o, e}$ are determined by the spectral resolution of $58 \mathrm{GHz}$ for samples \#1-3 and $29 \mathrm{GHz}$ for sample \#4. For sample \#1, the radius was taken from its SEM image (inset, Fig. 2a). *The radii of samples \#2 and \#4 were estimated using an optical microscope, which is less accurate as compared to SEM (+/-1um), and additionally adjusted within the error range via full-wave numerical simulations to match $\varepsilon_{o}=70$ (taken as a reference value from sample \#1). ${ }^{\dagger}$ The radius of sample \#3 was estimated using the value of the ordinary permittivity found for samples \#1,\#2, and \#4 via the full-wave simulations. It could not be measured as sample \#3 had fallen off the substrate and been lost. The errors in the radii of samples \#2-4 are linked to the spectral resolution of the experiment.

The quality factors $Q F_{o, e}$ vary from one sample to another. We attribute this to the morphology of the microspheres. SEM images and XRD analysis of the studied and similar spheres indicates the presence of voids and inclusions of other crystal phases, which affects the level of losses experienced by the excited magnetic dipole modes. The highest observed values of $Q F(\approx 90$, sample \# 4) are comparable with the best experimentally reported $Q F$ for aluminium split-ring resonators at $\mathrm{THz}$ frequencies [28-30].

Both the ordinary and extraordinary permittivities for all samples were extracted from numerical simulations, except for sample \# 3. For the latter, the ordinary permittivity of $\mathrm{TiO}_{2}$ was set equal to the value obtained for other samples and used to estimate the radius of the sample. The slight discrepancy in the values of the permittivities are most likely due to the material and geometrical variations of the measured $\mathrm{TiO}_{2}$ micro-spheres.

Fitting analytical Fano-resonance curves to our measured data confirmed the extreme narrowness - tens of gigahertz - of the observed magnetic dipole resonance peaks. This fact makes $\mathrm{TiO}_{2}$ micro-resonators a promising base for $\mathrm{THz}$ all-dielectric metamaterials and resonance-based devices, such as detectors and filters.

\section{Conclusions}

We detected the splitting of magnetic dipole resonances in anisotropic $\mathrm{TiO}_{2}$ spherical micro-resonators into ordinary and extraordinary modes using near-field $\mathrm{THz}$ timedomain spectroscopy. By changing the orientation of the samples with respect to the incident field polarization plane, we were able to excite either one of the modes or both of them simultaneously. We found that the near-field spectra are well described by the Fano-resonance model due to the underlying superposition of the modal fields and the incident standing wave formed behind the metallic screen of the near-field probe. By fitting the Fano-resonance model to the measured near-field spectra, we extracted the intrinsic properties of the studied anisotropic $\mathrm{TiO}_{2}$ micro-sphere resonators. We show, that one can describe the near-field spectra of the anisotropic dielectric resonators by a superposition of two Fano lineshapes, weighted in accordance to the orientation of the samples with respect to the polarization plane of the incident $\mathrm{THz}$ pulse. Using full-wave numerical simulations, we estimated the ordinary and extraordinary permittivities of mono-crystalline $\mathrm{TiO}_{2}$ as $\approx 70$ and $\approx 130$, respectively. Linewidths as narrow as tens of GHz in magnetic dipole resonances at $\approx 1 \mathrm{THz}$ suggest, that $\mathrm{TiO}_{2}$ micro-resonators are promising candidates for all-dielectric $\mathrm{THz}$ metamaterials, non-reflective metasurfaces based on Huygens sources, and resonance-based devices, including detectors and filters.

\section{Acknowledgements.}

This work is supported by the Royal Society [Grant No. UF130493], RFBR [16-07-01166 and 14-22-02064-ofi-m], the Czech Science Foundation (project 14-25639S), LabEx AMADEus (ANR-10LABX-42) in the framework of IdEx Bordeaux (ANR-10-IDEX-0302)/i.e./ the Investissements d Avenir programme of the French government managed by the Agence Nationale de la Recherche, and partially supported by the Government of the Russian Federation [Grant No. 074-U01 and GZ 3.561.2014/K]. This work was performed at UCL and, in part, at the Center for Integrated Nanotechnologies, a U.S. Department of Energy, Office of Basic Energy Sciences user facility. Sandia National Laboratories is a multi-program laboratory managed and operated by Sandia Corporation, a wholly owned subsidiary of Lockheed Martin Corporation, for the U.S. Department of Energy's National Nuclear Security Administration under contract DE-AC04-94AL85000.

Key words: terahertz spectroscopy, Mie scattering, dielectric metamaterials, magnetic dipole

\section{References}

[1] B. I. Popa and S. A. Cummer, Phys. Rev. Lett. 100, 207401 (2008).

[2] Q. Zhao, L. Kang, B. Du, H. Zhao, Q. Xie, X. Huang, B. Li, J. Zhou, and L. Li, Phys. Rev. Lett. 101, 027402 (2008).

[3] Q. Zhao, J. Zhou, F. Zhang, and D. Lippens, Mater. Today 12, 60-69 (2009). 
[4] J. C. Ginn, I. Brener, D. W. Peters, J. R. Wendt, J. O. Stevens, P. F. Hines, L. I. Basilio, L. K. Warne, J. F. Ihlefeld, P. G. Clem, and M. B. Sinclair, Phys. Rev. Lett. 108, 097402 (2012).

[5] S. Jahani and Z. Jacob, Nat. Nanotechnol. 11(1), 23-36 (2016).

[6] D. Lin, P. Fan, E. Hasman, and M. L. Brongersma, Science 345(6194), 298-302 (2014).

[7] M. Decker, I. Staude, M. Falkner, J. Dominguez, D. N. Neshev, I. Brener, T. Pertsch, and Y. S. Kivshar, Adv. Opt. Mater. 3(6), 813-820 (2015).

[8] A. Arbabi, Y. Horie, M. Bagheri, and A. Faraon, Nat. Nanotechnol. 10(11), 937-943 (2015).

[9] R. Merlin, Proc. Natl. Acad. Sci. U.S.A. 106(6), 16931698 (2008).

[10] L. Lewin, J. Inst. Electr. Eng. Part III Radio Commun. Eng. 94(27), 65-68 (1947).

[11] J. Geffrin, B. García-Cámara, R. Gómez-Medina, P. Albella, L. Froufe-Pérez, C. Eyraud, A. Litman, R. Vaillon, F. Gonzlez, M. Nieto-Vesperinas, J. Sáenz, and F. Moreno, Nat. Commun. 3, 1171 (2012).

[12] P. Spinelli, M. Verschuuren, and A. Polman, Nat. Commun. 3, 692 (2012).

[13] K. Baryshnikova, M. Petrov, V. Babicheva, and P. Belov, Sci. Rep. 6, 22136 (2016).

[14] B. Hopkins, D. S. Filonov, A. E. Miroshnichenko, F. Monticone, A. Al, and Y. S. Kivshar, ACS Photonics 2(6), 724 729 (2015).

[15] A. I. Kuznetsov, A. E. Miroshnichenko, Y. H. Fu, J. Zhang, and B. Lukyanchuk, Sci. Rep. 2, 492 (2012).

[16] H. Němec, C. Kadlec, F. Kadlec, P. Kužel, R. Yahiaoui, U.C. Chung, C. Elissalde, M. Maglione, and P. Mounaix, Appl. Phys. Lett. 100(6), 061117 (2012).

[17] O. Mitrofanov, F. Dominec, P. Kužel, J. L. Reno, I. Brener, U. C. Chung, C. Elissalde, M. Maglione, and P. Mounaix, Opt. Express 22(19), 23034-23042 (2014).

[18] K. Takano, Y. Yakiyama, K. Shibuya, K. Izumi, H. Miyazaki, Y. Jimba, F. Miyamaru, H. Kitahara, and M. Hangyo, IEEE Trans. Terahertz Sci. Technol. 3(6), 812819 (2013).

[19] R. Yahiaoui, K. Hanai, K. Takano, T. Nishida, F. Miyamaru, M. Nakajima, and M. Hangyo, Opt. Lett. 40(13), 3197-3200 (2015).

[20] C. Jördens, M. Scheller, M. Wichmann, M. Mikulics, K. Wiesauer, and M. Koch, Appl. Opt. 48(11), 2037-2044 (2009).

[21] A. J. Macfaden, J. L. Reno, I. Brener, and O. Mitrofanov, Appl. Phys. Lett. 104(1), 011110 (2014).

[22] I. Khromova, M. Navarro-Cía, I. Brener, J.L. Reno, a. Ponomarev, and O. Mitrofanov, Appl. Phys. Lett. 107(2), 021102 (2015).

[23] U. Fano, Phys. Rev. 124, 1866-1878 (1961).

[24] B. Luk'yanchuk, N. I. Zheludev, S. A. Maier, N. J. Halas, P. Nordlander, H. Giessen, and C. T. Chong, Nat. Mater. 9(9), 707-715 (2010).

[25] n.c.f. OriginLab, http://www.originlab.com/doc/ Origin-Help/Nonlinear-Curve-Fit/, 2000-2004.

[26] A. E. Miroshnichenko, A. B. Evlyukhin, Y. S. Kivshar, and B. N. Chichkov, ACS Photonics 2(10), 1423-1428 (2015).

[27] D. L. Markovich, P. Ginzburg, A. K. Samusev, P. A. Belov, and A. V. Zayats, Opt. Express 22(9), 10693-10702 (2014).
[28] W. Cao, R. Singh, I. A. I. Al-Naib, M. He, A. J. Taylor, and W. Zhang, Opt. Lett. 37(16), 3366-3368 (2012).

[29] L. Cong, M. Manjappa, N. Xu, I. Al-Naib, W. Zhang, and R. Singh, Adv. Opt. Mater. 3(11), 1537-1543 (2015).

[30] Y. K. Srivastava, M. Manjappa, L. Cong, W. Cao, I. AlNaib, W. Zhang, and R. Singh, Adv. Opt. Mater. 4(3), 457463 (2016). 